\title{
On/off Switch Mediated by Exo+ Polymerases: Experimental Analysis for Its Physiological and Technological Implications
}

\author{
Jia Zhang ${ }^{\dagger}$, Lin-Ling Chen ${ }^{\dagger}$, Zi-Fen Guo ${ }^{\dagger}$, Cui-Ying Peng ${ }^{\dagger}$, Duan-Fang Liao ${ }^{\dagger, *}$ and Kai Li ${ }^{\dagger,, \beta, *}$ \\ 'Genomapping Inc., Tianjin, Institute of SNP, Institute of Pharmacy and Pharmacology, Nanhua University, Hengyang 421001, China \\ ${ }^{\S}$ Institute of Life Science and Biotechnology, Jinan University, Guangzhou 510632, China
}

Received 3 April 2003, Accepted 11 June 2003

\begin{abstract}
The potential physiological role and technological application of the premature termination of DNA polymerization through the off-switch of exo+ polymerases were studied using $3^{\prime}$ phosphorothioate-modified or unmodified primers with single base mismatch distal to the 3' terminus. With exonuclease-digestible unmodified primers, a gradient premature termination of DNA polymerization was observed when amplified with exo+ polymerases. With $3^{\prime}$ allele specific phosphorothioatemodified primers, an efficient off-switch effect occurred in the discrimination of a single nucleotide polymorphism when directly using genomic DNA. Clearly, the off-switch of exo+ polymerases is useful in biomedical research.
\end{abstract}

Keywords: DNA polymerase, 3' Exonuclease, Primer extension, SNP

\section{Introduction}

In searching for a more practical technology for a SNP assay, we recently revealed the premature termination of DNA polymerization using 3 ' phosphorothioate-modified primers and exo+ polymerases, which was termed off-switch (Zhang and Li, 2003a, 2003b; Zhang et al., 2003c). We further suggested the potential physiological role of the off-switch in maintaining the fidelity in DNA replication and an immediate application in SNP screening. However, some questions need to be resolved. Can the on/off switch be observed in vitro with exonuclease-sensitive unmodified primers? Does this single base discrimination system work directly with the genomic DNA sample?

In this study, unmodified primers with a single base

\footnotetext{
*To whom correspondence should be addressed.

Tel: $858-549-8830$

Email: kaili34@yahoo.com or dfliao@hotmail.com
}

mismatch at different distances from the 3' end of primers, with or without phosphorothioate-modification, were employed to address these issues. We observed the premature termination of DNA polymerization with exonucleasesensitive primers and developed a practical assay for the genetic diagnosis of heritable deafness at a single base level, based on the on/off switch.

\section{Material and Methods}

Two types of DNA-dependent DNA polymerases were purchased from New England Biolab Inc. (Beverly, USA), Deep Vent and Deep Vent-. Deep Vent is the wild-type form that contains strong 3' to $5^{\prime}$ exonuclease activity. Deep Vent- is the form with a point mutation that results in the loss of proofreading function, which was used as the control.

Two-directional primer extensions were carried out with an annealing temperature of $56^{\circ} \mathrm{C}$ for $30 \mathrm{~s}$. Following denaturation at $95^{\circ} \mathrm{C}$ for two min, a primer extension was carried out for 30 cycles as follows: $30 \mathrm{~s}$ denaturation at $95^{\circ} \mathrm{C}, 30 \mathrm{~s}$ annealing, and $30 \mathrm{~s}$ extension at $72^{\circ} \mathrm{C}$. The primer extension reaction was performed in a total volume of $20 \mu \mathrm{l}$ with $10 \mathrm{pg}$ of the template for the short amplicon DNA and $10 \mathrm{ng}$ for genomic DNA as follows: $0.2 \mathrm{mM}$ $\mathrm{dNTP}, 0.01 \mathrm{u} / \mathrm{ml}$ of polymerase, $10 \mathrm{pmol} / \mathrm{ml}$ of both the sense and antisense primers, and $2 \mu \mathrm{l}$ of the $10 \times$ NEB polymerase reaction buffer, which provided a final concentration of $10 \mathrm{mM} \mathrm{KCl}, 20 \mathrm{mM}$ Tris- $\mathrm{HCl}\left(\mathrm{pH} 8.8\right.$ at $\left.25^{\circ} \mathrm{C}\right), 10 \mathrm{mM}\left(\mathrm{NH}_{4}\right)_{2} \mathrm{SO}_{4}, 2 \mathrm{mM} \mathrm{MgSO}{ }_{4}, 0.1 \%$ Triton X-100. The PCR products were visualized using $2 \%$ agarose gel electrophoresis running under $10 \mathrm{volt} / \mathrm{cm}$ in TBE.

Extension of $3^{\prime}$ exonuclease-sensitive primers The short amplicon set that was used for the unmodified primers was from Genomapping Inc. (Tianjin, China). These included four templates that differed from each other at a single nucleotide (Zhang et al., 2003a). The sense primer had the sequence of 5'-atccca agatatctgagaattc-3', which mismatched to point-mutated EcoR I sites residing in the templates. These are illustrated in Table 1 as follows: $-4 \mathrm{~mm}$ : gaTttc; $-3 \mathrm{~mm}$ : gaaAtc; $-2 \mathrm{~mm}$ : gaatAc; and -1 mm: gaattG. 
Table 1. Different results from unmodified mismatched primers extended by polymerase, with and without 3' exonuclease activity

\begin{tabular}{|c|c|c|c|c|}
\hline Template & Template sequences & $\begin{array}{l}\text { Polymerases used } \\
\text { of products }\end{array}$ & EcoR I digestibility & Sequencing data \\
\hline$-1 \mathrm{~mm}$ & atcccaagatatctgagaattG & Deep Vent- & + & ...gctgagaattctcagat... \\
\hline$-2 \mathrm{~mm}$ & atcccaagatatctgagaatAc & Deep Vent- & + & ...gctgagaattctcagat... \\
\hline$-3 \mathrm{~mm}$ & atcccaagatatctgagaaAtc & Deep Vent- & + & ...gctgagaattctcagat... \\
\hline$-4 \mathrm{~mm}$ & atcccaagatatctgagaTttc & Deep Vent- & + & ...gctgagaaatctcagat... \\
\hline$-1 \mathrm{~mm}$ & atcccaagatatctgagaattG & Deep Vent & - & ...gctgaCaattctcagat... \\
\hline$-2 \mathrm{~mm}$ & atcccaagatatctgagaatAc & Deep Vent & - & ...gctgagTattctcagat... \\
\hline$-3 \mathrm{~mm}$ & atcccaagatatctgagaaAtc & Deep Vent & - & ...gctgagaTttctcagat... \\
\hline$-4 \mathrm{~mm}$ & atcccaagatatctgagaTttc & Deep Vent & N/A & N/A \\
\hline
\end{tabular}

The antisense sequence is 5'-gaaacagctgaatggacccaa-3'. The capitalized nucleotide is the single base mismatch at the EcoR I site of the template.

Extension of $3^{\prime}$ ' exonuclease-resistant primers The human genomic DNA samples were phenol-chloroform extracted from 3 $\mathrm{ml}$ blood of two healthy volunteers. The primers that targeted the $\mathrm{C} \rightarrow \mathrm{T}$ point mutation at the GJB3 deafness gene (Xia et al., 1998) were designed with $3^{\prime}$ phosphorothioate-modification to render exonuclease resistance ( $\mathrm{Li}$ and Zhang, 2001). The sequence for the sense primer for $\mathrm{C}$ allele was as follows: 5 'caa cat cgt gga ctg cta cat tgc cc-3'; the antisense primer: 5'-gtg aag att ttc ttc ttg gta ggt cg-3'. The sense primer for the point mutated allele $\mathrm{T}$ was: $5^{\prime}$-caa cat cgt gga ctg cta cat tgc ct-3'; the antisense: $5^{\prime}$-gtg aag att ttc ttc ttg gta ggt ca-3'.

\section{Results}

Inefficient removal of mismatched nucleotide distal to the 3' terminus of exonuclease-digestible primers By using primers that had the EcoR I recognition sequence of GAATTC at the 3' end, efficient proofreading was quickly detected using EcoR I digestion. A sequencing analysis confirmed that the products from Exo- polymerase were EcoR
I-digestible since they inherited the wild-type sequence of the EcoR I site from primers, whereas the products that were amplified by exo+ polymerase were template-dependent and EcoR I-resistant (Table 1). This indicates the mismatch removal by 3 ' exonuclease of polymerase.

Products by exo+ polymerase were dramatically decreased with shifting mismatch away from the 3 ' terminus, which was different than a similar amount of products that were amplified by exo- polymerase (Fig. 1). When the single nucleotide mismatch was 3 bases away (-4) from the $3^{\prime}$ terminus, then no DNA polymerization occurred using exo+ polymerase, under the conditions used in this study. This data showed that the farther the mismatch was distal to the 3' terminus, then the mismatch removal occurred less efficiently. The data from the observed off-switch with exonucleasesensitive primers suggest a potential physiological role for the premature termination in DNA polymerization.

Single nucleotide discrimination using $\begin{array}{r}3^{\prime} \\ \text { phosphorothioate-modified allele-specific }\end{array}$ primers and
exo+ polymerases As a practical application of the novel

(A)
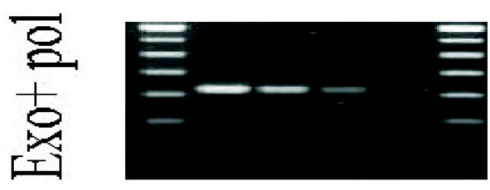

(B)
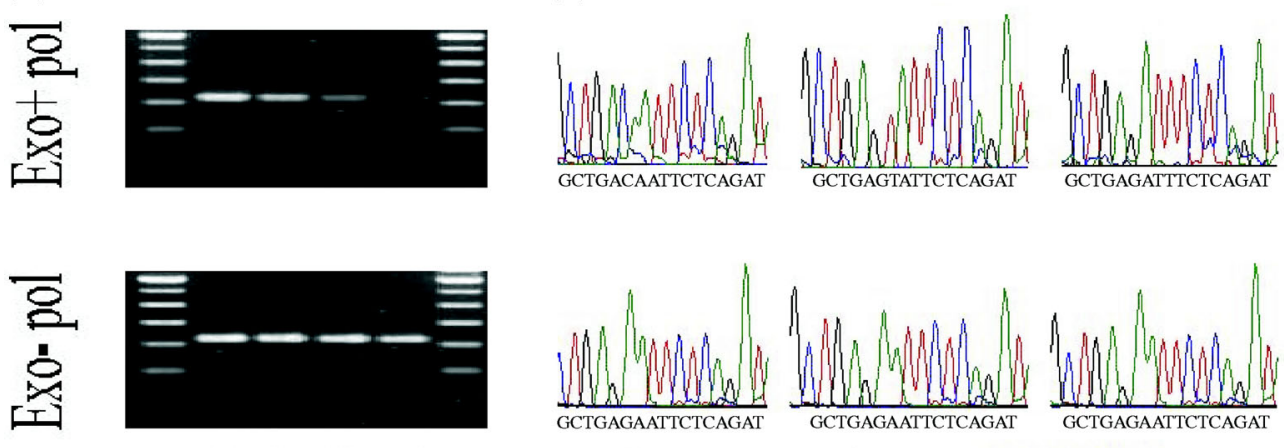

$-1-2-3-4$

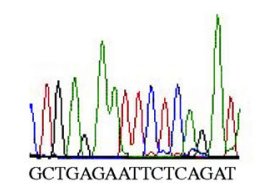

$-1$

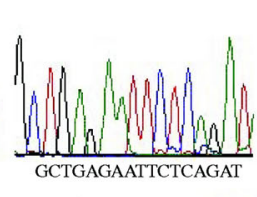

$-2$

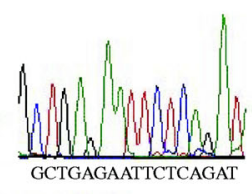

$-3$

Fig. 1. Suppression on DNA polymerization of exo+ pol by shifting the single base mismatch away from the primer-3'-terminus. When the single base mismatch shifted from -1 to -4 , the DNA polymerization by exo+ pol was gradually inhibited until completely turned off. The mismatch shifting had no effect on the primer extension by exo- pol. 


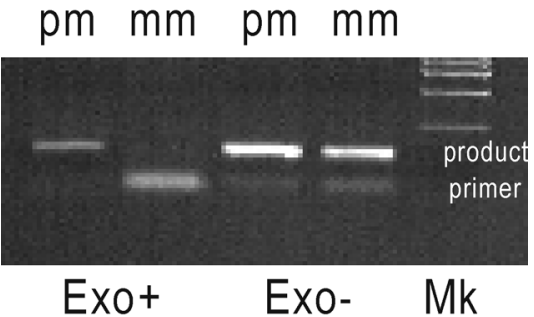

Fig. 2. The on/off switch that was operated by the PTO-modified primer-3'-termini targeting a recently identified $\mathrm{C} \rightarrow \mathrm{T}$ point mutation at the Gjb3 deafness gene. DNA templates identified from normal volunteers were CC homozygo, which only yielded products from primers for wild allele ( $\mathrm{pm}$ here). No product was amplified from the primer targeting $\mathrm{C} \rightarrow \mathrm{T}$ mutation ( $\mathrm{mm}$ here) by exo+ pol.

on/off switch, an assay targeting the recently-identified GJB3 deafness gene was developed. Two allele-specific primers, the sense and antisense primers, were used to target the wild or mutant allele of the GJB3 deafness gene. Using the genomic DNA from normal volunteers and exo+ polymerase (while the primers for normal allele were extended), no products were amplified from the 3' phosphorothioate modified primers that targeted the $\mathrm{C} \rightarrow \mathrm{T}$ point mutation at the GJB3 deafness gene (Fig. 2). This highly selective template-dependent DNA polymerization through the novel on/off switch insured the elimination of false positives in the clinical assay.

Similar to the results from the unmodified primers, exopolymerase that generated primer-dependent products from two types of the 3' phosphorothioate-modified allele specific primers (regardless of the homozygous templates) from healthy volunteers were used. As shown in Fig. 2, in this case polymerase without $3^{\prime}$ exonuclease activities failed to discriminate the template at the single base level.

\section{Discussion}

The fidelity maintenance through the off-switch of exo+ polymerases lacks a physiological basis since the phosphorothioate-modified 3' termini are not present in vivo. In the present study, we observed the on/off switch using more physiological 3' termini of unmodified primers with a single base that was mismatched, varying from one to several bases away from the 3 ' terminus. Furthermore, we also report the efficient off-switch effect with genomic DNA using the combination of 3' phosphorothioate-modified primers and exo+ polymerases.

In addition to the initial proofreading of the 3 ' terminal mismatch during DNA chain elongation, exo+ polymerases have the ability to proofread mismatches several bases upstream from the 3' termini in order to minimize mutations (Goodman and Fygenson, 1998; Zhang et al., 2003a). The successful excision of the upstream base mismatch is based on how far the mismatch is away from the $3^{\prime}$ terminus as well as the time that is required for excising a nucleotide. Using exopol lacking 3' exonuclease activities, the primer-dependent products were produced from amplicons with mismatches that were located on the end of the 3' terminus to 3' bases upstream, away from the $3^{\prime}$ terminus (Fig. 1). However, the template-dependent products that were amplified by exo+ polymerase decreased with the distance, where the mismatched nucleotide was distal to the 3 ' terminus. The most efficient mismatch removal was the 3 ' terminal mismatch. In the experimental condition that was used in this study, the -4 mismatch was inefficiently edited; it turned off the DNA polymerization by exo+ polymerase.

The inefficient excision by 3 ' exonuclease acts as an offswitch, which turns off DNA polymerization from mismatched primers. Exo+ pol uses this off-switch mechanism to maintain the high fidelity in DNA synthesis by truncating the products with mutations. Although the biological implication of this novel mechanism is unclear, we speculate that the premature termination of DNA synthesis by the off-switch plays a role in dividing the cells by ensuring mature products with minimum errors. For in vitro primer extension, this off-switch may provide a new avenue to genetic analysis. As shown in Fig. 2, a SNP that is associated with deafness was discriminated as a diagnostic assay: DNA polymerization was turned off from the primers that targeted the mutated allele. This encouraged the application of 3' PTOmodified primers and exo+ polymerases in the SNP assay and in the diagnosis for SNP-related genetic diseases.

As compared to most of the SNP assay, the on/off switch does not require prior amplification in the discrimination of SNP using genomic DNA samples. The requirement of prior amplification of the genes is a bottleneck that restrains the high throughput screening of known SNPs in the postgenome era (Zhang and Li, 2001; Bowtell and Sambrook, 2002; Zhang and Li, 2003a, 2003b). The present study directly demonstrates the ability of the newly identified on/off switch in the SNP analysis with the genomic DNA sample. The advantage of using genomic DNA samples in high throughput screening of a large number of SNPs is enormous. Prior amplification before single base discrimination is inconvenient and laborious but still possible for a single or limited amount of SNPs; however, it is technically difficult to achieve a high throughput screening of hundreds to thousands of different types of SNPs, either with microarray or microtiter technologies.

In conclusion, the data presented in this study suggests the potential physiological role of premature termination through the off-switch of 3' exonuclease of DNA polymerases. In addition, the template-dependent or allele-specific DNA polymerization using the novel on/off switch opened a new avenue to clinical diagnosis at the single nucleotide level.

Acknowledgments This study was partially supported by private funding from $\mathrm{Xi}-\mathrm{Kun} \mathrm{Li}$ to Genomapping Inc. during the period of Y2001-2002. LK is a recipient of a Guandong 
Provincial Grant (\#2002C5030601). LDF is a recipient of the National Major Basic Research Program of China (973: \#G2000056905).

\section{References}

Bowtell, D. and Sambrook, J. (2002) DNA Microarrays: A Molecular Cloning Manual, 1st ed., pp. 400-401, Cold Spring Harbor Laboratory Press, New York, USA.

Goodman, M. F. and Fygenson, D. K. (1998) DNA polymerase fidelity: from genetics toward a biochemical understanding. Genetics 148, 1475-1482.

Li, K. and Zhang, J. (2001) ISIS-3521 (ISIS Pharmaceutics). Curr. Opin. Investig. Drug 2, 1454-1461.

Xia, J. H., Liu, C. Y., Tang, B. S., Pan, Q., Huang, L., Dai, H.P., Zhang, B.R ., Xie, W., Hu, D. X., Zheng, D., Shi, X. L., Wang, D. A., Xia, K., Yu, K. P., Liao, X. D., Feng, Y., Yang, Y. F., Xiao, J. Y., Xie, D. H. and Huang, J. Z. (1998) Mutations in the gene encoding gap junction protein beta-3 associated with autosomal dominant hearing impairment. Nat.
Genet. 20, 370-373.

Zhang, J. and Li, K. (2001) The 3' terminal labeled primer extension: A new method of high throughput screening for SNP analysis. Curr. Drug Disc. 9, 21-24.

Zhang, J. and Li, K. (2003a) On/off regulation of 3' exonuclease excision to DNA polymerization by exo+ polymerase. $J$. Biochem. Mol. Biol. in press.

Zhang, J. and Li, K. (2003b) Single base discrimination mediated by proofreading $3^{\prime}$ phosphorothioate-modified primers. Mol. Biotechol. in press.

Zhang, J., Li, K., Deng, Z., Liao, D., Fang, W. and Zhang, X. (2003a) Efficient mutagenesis method for producing the templates of single nucleotide polymorphisms. Mol. Biotechol. 24, 105-110.

Zhang, J., Liao, D. F., Chen, L., Zhang, X. and Li, K. (2003b) Application of DNA polymerase with 3' exonuclease in SNP assay. J. Nanhua Univ. 31, 128-131.

Zhang, J., Li, K., Pardinas, J. R., Liao, D. F., Li, H. J. and Zhang, X. (2003c) SNP discrimination through proofreading and OFFswitch of exo+ polymerase. Mol. Biotechol. in press. 